\title{
Sui sistemi di funzioni analitiche e le serie formate coi medesimi.
}

\author{
(Prima Memoria di S. Pincherle, a Bologna.)
}

Lo studio delle serie di funzioni sferiche nel campo della variabile complessa conduce all'osservazione che molte proprietà di quelli sviluppi sono suscettibili di una generalizzazione molto ampia e tale da dare origine ad una speciale teoria, quella degli sviluppi in serie della forma

dove con

$$
\Sigma c_{n} p_{n}(x)
$$

$$
p_{n}(x) \quad(n=0,1, \ldots \infty)
$$

s'intende una determinata successione di funzioni analitiche. Una simile teoria può venir considerata sotto varî punti di vista, perchè varî sono i modi di definire una successione di funzioni: in questa prima Memoria ho tentato di trattare la questione in modo da conservare l'analogia colla teoria delle serie di funzioni sferiche.

Richiamerò dapprima quelle proprietà degli sviluppi in serie di funzioni sferiche la cui generalizzazione è sembrata maggiormente importante:

1. "La funzione sferica $p_{n}(x)$ di una variabile è definita come coeff"ciente di $y^{n}$ nello sviluppo di

$$
\left(1-2 x y+y^{2}\right)^{-\frac{1}{2}}
$$

$n$ in serje di potenze intere e positive di $y$.

"Essa funzione $p_{n}(x)$ ¿ un polinomio razionale intero di grado $n$ in $x$, e " come tale regolare per tutti i valori finiti di $x$.

$2 .^{\circ} "$ Uno sviluppo in serie di funzioni sferiche

$$
\sum c_{n} p_{n}(x)
$$

" converge entro un'ellisse descritta nel piano della variabile $x$ coi fuochi nei 
"punti 1 e -1 . I semi assi di questa ellisse sono

$$
\frac{1}{2}\left(\rho+\frac{1}{\rho}\right), \quad \frac{1}{2}\left(\rho-\frac{1}{\rho}\right)
$$

$"$ se $\rho$ è il raggio di convergenza della serie $\mathbf{\Sigma} c_{n} x^{n}$. Le ellissi del sistema "omofocale coi fuochi \pm 1 sono per le serie di funzioni sferiche, ciò che le " circonferenze col centro nell' origine sono per le serie di potenze. Nei campi " chiusi da linee di questo sistema le serie di funzioni sferiche godono della " convergenza incondizionata ed in egual grado.

$3 .^{\circ}$ "Al sistema delle funzioni sferiche $p_{n}(x)$ si può associare un secondo $n$ sistema di funzioni $q_{n}(y)$ dette $d i$ seconda specie; queste, limitando conve" nientemente il campo delle variabili $x$ ed $y$, danno luogo all'identità

$$
\frac{1}{y-x}=p_{n}(x) q_{n}(y)
$$

" da cui e dal toorema di Cavcry si deduce che ogni funzione analitica $f(x)$ "regolare entro un'ellisse di fuochi \pm 1 è sviluppabile in serie di funzioni $"$ sferiche $p_{n}(x)$, e lo sviluppo converge incondizionatamente ed in egual grado "entro l'ellisse, il contorno escluso. Una funzione analitica regolare fuori di " una simile ellisse è sviluppabile invece in serie di funzioni di seconda specie, "ed una funzione regolare nell' anello compreso fra due ellissi omofocali è svi"luppalile in serie di funzioni $p_{n}(x)$ e $q_{n}(x)$.

"Inoltre lo sviluppo di una funzione analitica in serie di $p_{n}(x) \quad q_{n}(x)$ è $"$ possibile in un sol modo, ciod non esistono sviluppi dello zero in serie di "funzioni sferiche.

4. "Le proposizioni precedenti permettono di risolvere il seguente pronblema: dato un sistema di punti

$$
a_{n} \quad(n=0, \ldots \infty)
$$

"nel piano della variabile $x$ ed i punti limiti del sistema $a_{n}$ trovandosi sopra $"$ un' ellisse, costrurre una funzione analitica che nei punti $a_{n}$ ammetta singo"larità di specie determinata, ed in particolare costruire una funzione analitica " che abbia uno spazio lacunare (interno od esterno) di forma ellittica $(*)$."

La presente Memoria ha per oggetto la generalizzazione delle proprietà ora ricordate. Nel primo capitolo si premettono alcune definizioni e proposizioni

() Per le proprieta $2^{n}$ e $3^{\mathrm{h}}$, v. Neumans (Halle, 1862) e 'Thomé (J. de Cremriz, t. 66); per la $4^{2}$, $v$. una mia Nota nei Rendiconti dell' Accademia di Bologna, marzo 1883. 
generali sui gruppi di numeri ed i sistemi di funzioni, nel secondo si indica un modo generale di ottenere sistemi di funzioni analitiche, e si cerca quali siano le linee che limitano i campi di convergenza delle serie procedenti per tali funzioni. Nel terzo si suppone che ad un dato sistema di funzioni $p_{n}(x)$ corrisponda un altro sistema, che si dirà associato; si cerca, mediante il teorema di Caucrix, quali funzioni siano sviluppabili in serie di funzioni $p_{n}(x)$, e si mostra come sotto certe condizioni si conservino in questi sviluppi le proprietà riscontrate nelle serie di funzioni sferiche. Infine gli ultimi due capitoli sono dedicati allo studio di un metodo che si può, entro certi limiti, sostituire al teorema di $\mathrm{C}_{A U C H Y}$ e che si presenta in qualche modo come una estensione della teoria delle equazioni lineari in casi in cui il numero delle equazioni e delle incognite diventa infinito.

Una seconda Memoria tratterà dello studio degli sviluppi dello zero, che non vengono considerati in questo primo lavoro, e all'esame della questione: se, dato un sistema di funzioni, si possa dire se esso ammette o no un sistema associato e nel caso affermativo, in qual modo si possa giungere a determinarlo.

I.

1. Un sistema di numeri reali o complessi in numero infinito

$$
a_{n} \quad(n=0,1, \ldots \infty)
$$

verrà detto un gruppo ed indicato con $\left(a_{n 2}\right)$.

Un insieme $\infty^{\infty}$ di gruppi verrà detto una varietà.

Un gruppo $\left(a_{n}\right)$ si dirà interno alla varietà $r$, indicando $r$ un numero positivo, se si potrà determinare un numero positivo $r^{\prime}<r$ tale che da un valore di $n$ in avanti, sia sempre

$$
\left|a_{n}\right| \leq C r^{\prime n}
$$

dove $C$ è un numero positivo finito ed indipendente da $n$.

Un gruppo $\left(a_{n}\right)$ si dirà esterno alla varietà $r$ se si potrà determinare un numero positivo $r^{\prime \prime}>r$ e tale che per infiniti numeri $a_{n}$ del gruppo si abbia

$$
\left|a_{n}\right| \geqslant C r^{\prime \prime} \text {. }
$$

Risulta evidentemente da queste definizioni che se un gruppo $\left(a_{n}\right)$ è interno alla varietà $r$, è interno altresì a tutte le varietà $r^{\prime \prime}$ maggiori (per le quali $r^{\prime \prime}$ $\grave{\text { è }}>r$ ) e che se il gruppo è esterno alla varietà $r$, ̀̀ esterno a tutte le varietà minori. 
2. Se un gruppo $\left(a_{n}\right)$ non è nè interno nè esterno ad una varietà $r$, esso si dirà sul contorno della varietà stessa; così i gruppi

$$
\text { (n), }\left(\frac{1}{n}\right), \quad\left(\frac{n-1}{n-1}\right) \text {, }
$$

sono sul contor'no della varietà $1\left(^{*}\right)$. Questa definizione è giustificata dalle considerazioni che seguono.

Se il gruppo $\left(a_{n}\right)$ è sul contorno della varietà $r$ esso non può essere esterno ad alcuna varietà maggiore, nè interno ad alcuna varietà minore.

Se la serie $\Sigma_{n} a^{n}$ ammette il raggio effettivo di convergenza $R$, il gruppo $\left(a_{n}\right)$ è sul contorno della varietà $\frac{1}{R}$ : infatti esso non è interno, poichè si avrebbe per $\frac{1}{i}<\frac{1}{n}$

$$
\left|a_{n}\right| \leq \frac{C}{r^{n}}
$$

ossia la seric convergerebbe in un cerchio di raggio $r>R$, contro l'ipotesi, nè esterno, poichè si avrebbe per infiniti valori di $n$ e per $r^{\prime}<R$

$$
\left|a_{n}\right| \geq \frac{O}{r^{\prime} \cdot}
$$

il che non può essere, come dalla teoria delle serie di potenze. Inversamente, se il gruppo $\left(a_{n}\right)$ è sul contorno della varietà $r$, la serie $\mathbf{Z} a_{n} x^{n h}$ ha per cerchio effettivo di convergenza il cerchio di raggio $\frac{1}{r}$; infatti essa non ha un cerchio di raggio maggiore $\frac{1}{\rho}>\frac{1}{r}$ perchè dovrebbe essere per $\frac{1}{\rho}>\frac{1}{\xi}>\frac{1}{r}$

$$
\left|a_{n}\right| \leq C \xi^{n}
$$

cioè $\left(a_{n}\right)$ sarebbe interno alla varietà $r$, nè minore, perchè per $r^{\prime}$ maggiore di $r$ di tanto poco quanto si vuole deve essere

$$
\left|a_{n}\right| \leq C r^{\prime} n
$$

che dimostra che $\mathbf{\Sigma} a_{2 \imath} x^{n}$ converge entro il cerchio $\frac{1}{\gamma^{*}}$.

Risulta da questo teorema che uno stesso gruppo non può trovarsi contem-

(c) Una scrie $\mathbf{\Sigma} a_{n}$ è convergente incondizionatamente se i suoi termini tormano un gruppo $\left(a_{n}\right)$ interno alla varieta 1 , è divergente so formano un gruppo esterno, vi d dubbio se il gruppo ò sul contorno della varieta 1 . 
poraneamente sul contorno di due diverse varietà, e che se il gruppo $\left(a_{n}\right)$ è sul contorno della varietà $r$, esso è interno ad ogni varietà maggiore ed esterno ad ogni varietà minore di $r^{\circ}$.

3. Se un gruppo $\left(a_{n}\right)$ è tale che preso un numero positivo $\varepsilon$ arbitrariamente piccolo sia, a partire da un certo valore dell'indice $n$,

$$
\left|a_{n}\right| \leq C \hat{e}^{n},
$$

il gruppo sarà interno a qualunque varietà, per quanto piccola, e si potrà dire gruppo ologene, perchè dà origine, mediante la serie $\mathbb{\Sigma} a_{n} x^{n}$, ad una funzione intera.

Se un gruppo $\left(a_{n}\right)$ è tale che preso un numero positivo $M$ arbitrariamente grande, vi siano infiniti termini del gruppo tali che sia

$$
\left|a_{n}\right| \geqslant C M^{n}
$$

il gruppo sarà esterno a qualunque varietà, per quanto grande, e si potrà dire al contorno della varietà $\infty$; in tal caso la $\sum a_{n} x^{n}$ non è mai convergente.

Per ogni altro gruppo $\left(a_{n}\right)$ esisterà un numero determinato $r$ che sarà l'inverso del raggio di convergenza della serie $\sum_{n} x^{n}$, e tale che il gruppo si trova sul contorno della varietà $r$.

4. Se due gruppi $\left(a_{n}\right)$ e $\left(b_{n}\right)$ sono, l'uno interno alla varietà $r$, l'altro interno o sul contorno della varietà $r^{\prime} \geq r$, il gruppo $\left(a_{n} \pm b_{n}\right)$ sarà interno alla varietà $r^{\prime}$, o sul contorno.

Se due gruppi $\left(a_{n}\right)$ e $\left(b_{n}\right)$ sono interni rispettivamente alle varietà $r$ ed $r$, i) gruppo $\left(a_{n} b_{n}\right)$ sarà interno alla varietà $r r^{2}$; se i gruppi $\left(a_{n}\right)$ e $\left(b_{n}\right)$ sono sul contorno delle varietà $r$ ed $r^{\prime}$, il gruppo $\left(a_{n} b_{n}\right)$ sarà interno ad ogni varietà maggiore di $r r^{\prime}$, ma non si potrà asserire che sia esterno ad ogni varietà minore e perciò non si potrà dire sul contorno della varietà $r r^{\prime}$; talchè se $R$ ed $R^{\prime}$ sono i raggi di convergenza di $\sum a_{n} x^{n}$ e $b_{n} x^{n}, \sum_{n} b_{n} x^{n}$ arrà il raggio di convergenza non minore di $R E$.

Se $\left(a_{n}\right)$ è interno alla varietà $r,\left(\frac{1}{a_{n}}\right)$ sarà esterno alla varietà $\frac{1}{r}$ e se $\left(a_{n}\right)$ è sul contorno della varietà $r,\left(\frac{1}{a_{n}}\right)$ sarà esterno ad ogni varietà minore di $\frac{1}{r}$.

5. Se un sistema di funzioni ad un valore, in numero infinito

$$
\varphi_{n 2}(x) \quad(n=0,1, \ldots \infty)
$$

dà origine per ogni valore $x$ contenuto entro un campo $A$ ad un gruppo posto nell'interno o sul contorno della varietà $r$, si dirà che il sistema di funzioni è, per quei valori di $x$, rispettivamente interno o sul contorno della varietà. 
Se un sistema di funzioni $\varphi_{n}(x)$ è interno alla varietà $r$ pei valori di $x$ contenuti in un campo $A$, la serie $\sum c_{n} \varphi_{n}(x)$ sarà convergente assolutamente entro il campo $A$ se il gruppo $\left(c_{n}\right)$ è interno alla varietà $\frac{1}{r}$ o sul contorno.

Se un sistema di funzioni $\varphi_{n}(x)$ è tale che per tutti i valori di $x$ contenuti in un campo $A$ entro il quale le funzioni rimangono tutte finite, ed escluso il punto $x=0$, il quoziente

$$
\frac{\varphi_{n}(x)}{x^{n}}
$$

sia sempre sul contorno di una varietà $r$, la serie $\mathbf{\Sigma} c_{n} \varphi_{n}(x)$ converge e diverge rispett. nei punti del campo $A$ interni od esterni al cerchio di convergenza di $\Sigma c_{n} r^{m n} x^{n}$. In particolare se il quoziente $\frac{\rho_{n}(x)}{x^{n}}$ si trova al contorno della varietà 1 , la serie $\sum c_{n} \varphi_{n}(x)$ converge e diverge rispett. nei punti del campo $A$ interni od esterni al cerchio di convergenza di $\sum c_{n} x^{n}$.

II.

6. Sia

$$
T(u, v)
$$

una funzione analitica di due variabili che per ogni coppia $\left(u_{0} v_{0}\right)$ di valori delle variabili sia regolare (cioè sviluppabile in serie della forma

$$
\underset{m, n}{\sum_{m, n}} c_{m, n}\left(v-v_{0}\right)^{m}\left(u-u_{0}\right)^{n}
$$

per valori di $\left|u-u_{0}\right|,\left|v-v_{0}\right|$ abbastanza piccoli) eccettuate le coppie soddisfacenti ad un' equazione

$$
f(u, v)=0
$$

dove si suppone $f(u, v)$ funzione intera di $u$ e $v$. Per $v=0$ la funzione (1) sarà regolare per tutti i valori di $u$, tranne quelli che soddisfanno all' equazione

$$
f(u, 0)=0
$$

e che saranno punti separati

$$
0_{1}, \quad 0_{2}, \quad 0_{3}, \ldots \quad 0_{n}, \ldots
$$

del piano $u$, tali che entro un cerchio di raggio finito se ne trovi sempre solo un numero finito. Se $u_{0}$ è un punto che non sia radice della (3), si avrà

$$
T(u, v)=\sum_{n, n} a_{m, n}\left(u-u_{0}\right)^{n} v^{m}
$$


e questo sviluppo, per valori di $\left|u-u_{0}\right|$ e di $|v|$ abbastanza piccoli, converge incondizionatamente, per cui si può ordinare rispetto alle potenze di $v$ ed il coefficiente di $v^{m}$ sarà

$$
\sum_{n=0}^{\infty} a_{m, n}\left(u-u_{0}\right)^{n}
$$

che converge, qualunque sia l'indice $m$, entro un cerchio di centro $u_{0}$ e di raggio determinato.

Ora è noto $\left(^{*}\right)$ che la serie (5) costituisce un elemento di una funzione analitica che diremo $p_{m}(u)$; questa funzione è pienamente definita dal suo elemento ed $\mathrm{i}$ suoi valori si possono dedurre dalla serie (5) per ogni punto del campo di validità della funzione: in altri termini, per ogni punto $\vec{u}$ dell'interno del suo campo di validità $p_{m}(u)$ è sviluppabile in una serie di potenze di $u-\bar{u}$ che si può ricavare, con processo conosciuto, dalla serie (5). $\mathrm{Ma}$ preso un punto $u_{1}$ entro il campo di convergenza della serie (4), si avrà d'una parte, indicando con $q_{m}\left(u-u_{1}\right)$ la serie dedotta immediatamente dalla (5) relativamente al punto $u_{1}$ :

$$
T(u, v)=\Sigma v^{m} \eta_{m}\left(u-u_{1}\right)
$$

per valori di $\left|u-u_{1}\right|$ abbastanza piccoli; d'altra parte, dallo sviluppo (4) di $T(u, v)$ relativo al posto $\left(0, u_{0}\right)$ si potrà dedurre uno sviluppo relativo al posto $\left(0, u_{1}\right)$, della forma

$$
T(u, v)=\mathbf{\Sigma} a_{m, n}^{\prime} v^{m}\left(u-u_{1}\right)^{n}
$$

e poichè questo, per valori di $\left|u-u_{1}\right|$ abbastanza piccoli, converge incondizionatamente, si avrà ordinando per le potenze di $v$ :

$$
T(u, v)=\mathbf{E} v^{m}{ }_{x_{i}^{\prime}}^{\prime}\left(u-u_{1}\right)
$$

e per teoremi noti sulle serie di potenze, $\mathfrak{P}_{m}^{\prime}$ non potrà differire da $\mathfrak{P}_{m}$. Se ora si ragiona in modo analogo per un punto $u_{2}$ dell'intorno di $u_{1}$, indi per un punto $u_{3}$ dell'intorno di $u_{2}$, ecc., si giunge alla conclusione che:

"Per ogni punto del piano $u$ vale l'eguaglianza

$$
T(u, v)=\mathbf{\Sigma} v^{m} p_{m}(u)
$$

$n$ tolti i soli punti $0_{n}$; dove le funzioni $p_{m}(u)$ definite da un loro elemento (5),

(') Per la nomenclatura quì usata, v. WeIERstrass: Zur Functionenlehre (Monatsbericht der Berl. Akad.) e suo corso litografato di lezioni: Sulle funzioni di piu variabili (Berlino, presso Hermann) od anche il mio Saggio di una Introduzione alla teoria delle funzioni analitiche secondo $i$ principt di $C$. Weierstrass (Giomale di BATtagun, t. 18). 
$n$ sono regolari in tutto il piano, eccettuati al più gli stessi punti $0_{n}$. Ed il " valore da assegnarsi ad ogni funzione $p_{m}(u)$ in un punto $\bar{u}$ del piano $u$ che "non sia uno dei punti $0_{n}$ è dato dal coefficiente di $v^{m}$ nello sviluppo di $T(\bar{u}, v)$ "per le potenze intere e positive di $v$."

Se l'equazione (3) non ha soluzioni finite, le funzioni $p_{m}(u)$ sono intere.

La fanzione $T(u, v)$ si dirà funzione generatrice del sistema di funzioni $p_{m}(u)$.

7. Indichiamo con $A$ il campo che si ottiene togliendo dal piano $u$ i punti $0_{n}$, e fissiamo entro $A$ un punto qualunque $u_{0}$ : per esso si avrà

$$
T\left(u_{0}, v\right)=\underset{s=d}{v} v^{m} p_{m}\left(u_{0}\right)
$$

e la serie del secondo membro convergerà entro un cerchio posto nel piano $v$ col centro 0 e di raggio determinato; ma siccome la convergenza della serie non può cessare che in una circonferenza sulla quale si trovi qualche punto singolare della funzione, così il raggio del suddetto cerchio "sarà eguale al " minimo valore assoluto delle radici dell' equazione

$$
f\left(u_{\mathrm{s}}, v\right)=0 . "
$$

8. Prendiamo dunque a considerare la corrispondenza stabilita fra $\mathrm{i}$ punti dei due piani $u$ e $v$ dalla relazione

$$
f(u, v)=0 \text {. }
$$

Ad ogni valore $u_{0}$ di $A$ corrisponde per questa equazione un sistema di valori di $v$, i cui valori assoluti

$$
\left|v_{0}\right|, \quad\left|v_{0}^{\prime}\right|, \quad\left|v_{0}^{\prime \prime}{ }_{0}^{1}, \ldots \quad\right| v_{0}^{n} \mid, \ldots
$$

non possono avere altro posto limite fuorchè l' $\infty$ per la forma della funzione $f(u, v)$; per cui ve ne sarà uno minimo ed inoltre diverso da zero, perchè $u_{0}$ è interno al campo $A$. Si denoti con $\rho\left(u_{0}\right)$ questo minimo. Al variare di $u_{0}$, varierà pure la quantità definita con $\rho\left(u_{0}\right)$ e posto $u=\xi+i \eta, \rho(u)$ sarà funzione di $\xi$ ed $n$ nel senso generale ed anche, come risulta dai principî della teoria delle funzioni, sarà funzione continua; ad ogni valore di $u$ corrisponde inoltre un valore di $\rho(u)$ ed uno solo. Se fissiamo un valore o, e cerchiamo tutti i punti del piano $u$ per i quali

$$
p(u)=\alpha,
$$

linsieme di questi punti costituirà generalmente una linea che diremo curva $C_{\alpha}$ e che sarà composta di uno o più rami di curva analitica: più precisamente, essa sarà parte di una fra quelle curve che nel piano $u$ corrispondono mediante 
la rappresentazione (2) ai cerchi descritti nel piano $v$ dall'origine come centro; infine, per l'osservazione fatta dianzi, le $C_{\alpha}$ si deformeranno con continuità al variare con continuità di $\alpha$, e per ogni punto del campo $A$ passerà una curva $C_{\alpha}$ ed una sola. Però non esisteranno sempre, per tutti i valori a positivi e diversi da zero, punti $u$ nei quali sia $\rho(u)=\alpha$, ossia non tutti i valori $\alpha$ saranno possibili come valori assoluti minimi delle radici delle equazioni (2), e quindi non tutti i cerchi $\alpha$ si possono far corrispondere alle linee definite con $C_{\alpha}$. Infatti se qualunque valore $\alpha$ fosse possibile, esso potrebbe prendersi grande quanto si vuole, cioè dovrebbero esistere valori $\bar{u}$ di $u$ per i quali l'equazione

$$
f(\bar{u}, v)=0
$$

arrebbe tutte le sue radici infinite, il che non arviene che in casi particolari, qualora $f(u, v)$ prenda la forma $e^{G(v)}$, oppure, posto

$$
f(u, v)=\mathbf{\Sigma} v^{m} f_{m}(u),
$$

quando il valore $\bar{u}$ sia tale da annullare tutte le $f_{m}(u)$, ad eccezione della $f_{0}(u)$. Risulta da ciò e dalla continuità di $\rho(u)$, che i valori possibili di $\alpha$ saranno compresi fra 0 ed un numero $R$, che non è infinito se non per forme speciali della $f(u, v)$.

I soli punti del piano $u$ ai quali possa corrispondere un cerchio di raggio 0 sono $\mathrm{i}$ punti $0_{n}$, talchè il sistema dei punti (discreti) $0_{n}$ si potrà riguardare come la curva $C_{0}$.

9. Fissato un valore $\alpha$ fra quelli possibili, si potranno considerare nel campo $A$ due regioni: la prima, che si dirc̀ $E_{a}$, per il punto della quale sia $\rho(u)>\alpha$, l'altra $E_{\alpha}^{\prime}$, nei cui punti sia $\rho(u)<\alpha$. Queste regioni constano di aree connesse o no, che ricoprono una sol volta il campo $A$ e sono separate dalla linea $C_{a}$.

Consideriamo ora la serie

$$
\Sigma v^{m} p_{m}(u)
$$

per un punto $u$ posto sulla linea $C_{\alpha}$, essa converge entro il cerchio di centro 0 e di raggio a del piano $v$; per un punto $u$ preso nella regione $\boldsymbol{L}_{\alpha}^{2}$, essa converge entro un cerchio concentrico di raggio maggiore. Se dunque si fa $v=\alpha$, si ottiene la serie

$$
\mathbf{\Sigma}_{\alpha^{m}} p_{m}(u)
$$

che convergerà certamente per tutti i valori $u$ presi nell' interno della regione $E_{\alpha}$, e poichè la serie $\Sigma v^{m} p_{m}(w)$ considerata come serie di potenze di $v$, con- 
verge incondizionatamente entro il suo cerchio di convergenza, cosi la (7) convergerd incondizionatamente entro il campo $E_{*}$.

Si considerino ora le coppie $(u, v)$ tali che $u$ sia interno ad $E_{\alpha}$ e $v$ al cerchio $\alpha$ : per queste coppie la serie (6) non può cessare di convergere poichè $u$ è preso in modo che sia $\rho(u)>\alpha$, e se si prende un'area $\widetilde{E}_{\alpha}$ interna ad $E_{\alpha}$ ed un cerchio $\alpha$ minore di $\alpha \theta$ differente da $\alpha$ di tanto poco quanto si vuole, per le coppie $(u, v)$ tali che $u$ sia interno ad $\overline{E_{a}}$ e $v$ al cerchio $\alpha^{\prime} \mathrm{i}$ valori della serie (6) avranno un limite superiore $L$. Sia ora un numero $\alpha^{\prime \prime}>\alpha$, d'altronde differente da $\alpha$ di tanto poco quanto si vuole: il campo $E_{\alpha}$ " differirà di pochissimo da $E_{\alpha}$ e per tutti i punti $u$ comuni ai campi $\bar{E}_{o s}$ ed $E_{\alpha^{\prime \prime}}$, si avrà

$$
\left|p_{m}(u)\right|<\frac{L}{\alpha^{\prime \prime m}}
$$

da cui

il che dimostra:

$$
\left|\mathbf{Z} \alpha^{m} p_{m}(u j)\right|<L \mathbf{\Sigma}\left(\frac{\alpha}{\alpha^{u}}\right)^{m}
$$

" Che pei valori di a compresi fra 0 ed $R(\S 8)$, le serie (7) convergono " incondizionatamente entro $\mathrm{i}$ campi indicati con $E_{\alpha}$ e limitati dalle curve $C_{\alpha}$, " e che in un campo qualunque interno ad $E_{\alpha}$ esse convergono in egual grado "e sono quindi suscettibili di rappresentare funzioni analitiche di $u_{\text {. }}$.

Non si può dire nulla di generale sul modo di comportarsi delle serié (7) lungo le curve $C_{\alpha}$; entro $\mathrm{i}$ campi $E_{\alpha}^{\prime}$ esse divergono.

10. Sia $\left(c_{m}\right)$ un gruppo di numeri posto al contorno della varietà $\propto(\S 2)$ : si avrà, essendo $\varepsilon$ un numero positiro arbitrariamente piccolo ed $H$ un numero positivo finito:

e quindi

$$
\left|c_{m}\right|<H(\alpha+\varepsilon)^{n}
$$

$$
\left|\mathbf{\Sigma} c_{m} p_{m}(u)\right|<H \mathbf{\Sigma}(\alpha+\varepsilon)^{m} \cdot\left|p_{m}(\boldsymbol{u})\right|
$$

ma la serie del secondo membro converge entro il campo $E_{\alpha+\varepsilon}$, onde lo stesso sard̀ di $\mathbf{\Sigma} c_{m} p_{m}(u)$, e poichè $\varepsilon$ è arbitrariamente piccolo e le curve $C_{\alpha}$ si deformano con continuità al variare di $\alpha$, la $\boldsymbol{\Sigma} c_{m} p_{m}(u)$ convergerà incondizionatamente in tutto l'interno di $E_{\alpha}$. Mediante il ragionamento fatto nel paragrafo precedente, se ne dimostrerebbe ancora la convergenza in egual grado in qualunque campo interno ad $E_{\alpha}$, per cui:

¿Le proprietà delle serie (7) espresse alla fine del paragrafo precedente si 
" conservano nelle serie più generali

$$
\Sigma c_{m} p_{m}(u)
$$

ndove $\left(c_{m}\right)$ è un gruppo al contorno della varietà $\alpha_{.}$"

11. Le curve $C_{\alpha}$ ed i campi di convergenza $E_{\alpha}$ determinati in ciò che precede, sono indipendenti dalla natura della funzione generatrice $T(u, v) \mathrm{e}$ dipendono unicamente dalla equazione $f(u, v)=0$ che ne dà i posti singolari. Risulta pure dalle cose dette che se $\alpha^{\prime}>\alpha$, tutti i punti di $E_{\alpha}$ appartengono ad $E_{\alpha}$ e siccome essi non possono coincidere $(\S 8)$ così $E_{\alpha}$ deve essere maggiore di $E_{\alpha^{\prime}}$.

12. Se per un sistema di funzioni $p_{m}(u)$ sono determinati i campi di convergenza $E_{a}$, ed un secondo sistema $\varpi_{m}(u)$ è tale che per ogni valore di $u$, esclusi al più punti discreti, il gruppo

$$
\left(\frac{\varpi_{m}(u)}{p_{m}(u)}\right)
$$

sia sul contorno della varietà 1 , le serie

$$
\mathbf{\Sigma} c_{m} \varpi_{m}(u)
$$

convergeranno negli stessi campi delle

$$
\sum_{i=d} c_{m} p_{m}(u) \text {. }
$$

Infatti, preso $\varepsilon$ positivo e piccolo quanto si vuole, si avrà

onde

$$
\left|\varpi_{m}(u)\right|<(1+\varepsilon)^{m}\left|p_{m}(u)\right|
$$

$$
\left|\Sigma c_{m} \boldsymbol{\omega}_{m}(u)\right|<\Sigma\left|c_{m}\right|(1+\varepsilon)^{m}\left|p_{m}(u)\right|
$$

e se $c_{m}$ è sul contorno della varietà $\alpha$, jl secondo membro e quindi anche il primo convergeranno entro il campo $E_{\alpha(1+\varepsilon)}$, e poichè $\varepsilon$ è piccolo quanto si vuole, la

$$
\Sigma c_{m} \varpi_{m}(u)
$$

convergerà (eziandio incondizionatamente) entro il campo $E_{q}$.

Inversamente, se è tracciato nel piano il sistema delle anzidette curve $C_{\alpha}$, ognuna delle quali separi le due regioni $E$ ed $E_{\alpha}^{\prime}$, e se due sistemi di funzioni $p_{m}(u)$ e $\omega_{m}(u)$ singolari solo nei punti della $C_{0}$ sono tali che le serie

$$
\mathbf{\Sigma} \alpha^{m} p_{m}(u) \text { e } \quad \mathbf{\Sigma} \alpha^{m} \varpi_{m}(u)
$$

convergono incondizionatamente in $E_{\alpha}$ e divergono in $E_{\alpha^{\prime}}$, il gruppo

$$
\left(\frac{p_{m}(u)}{\varpi_{m}(u)}\right)
$$


sarà per ogni valore di $u$, eccettuati quelli sulla $C_{0}$, sul contorno delle varietà 1. Infatti, se fosse al contorno di una varietà minore $r$, si avrebbe, preso

onde

$$
\begin{gathered}
1>r^{\prime}>r: \\
\left|p_{n n}(u)\right|<r^{\prime}{ }^{m}\left|\varpi_{m}(u)\right|
\end{gathered}
$$

$$
\underline{\Sigma} \alpha^{m} \mid p_{m}(u)_{i}^{\prime}<\mathbf{\Sigma}_{-1} \alpha_{m} r^{\prime m} \omega_{m}(u)
$$

ora $E_{\alpha r^{\prime}}$ per $r^{\prime}<1$, ̀̀ maggiore di $E_{\alpha}$, cioè contiene anche punti di $E_{\alpha}^{\prime}$; se quindi $u$ è un punto comune ad $E_{\alpha}^{\prime}$ e $E_{\alpha r}$, la serie del 2 ' membro converge e quindi a maggior ragione quello del primo, il che è contro l'ipotesi. Analogamente se il gruppo fosse al contorno di una varietà maggiore d'uno, si considererebbe

$$
\left(\frac{\varpi_{m}(u)}{p_{n}(u)}\right)
$$

e si ripeterebbe lo stesso ragionamento.

Ritenendo una notazione usata da Frebenits (*) in circostanza analoga, ma più particolare assai, potremo scrivere

$$
\varpi_{m}(u) \sim p_{m}(u)
$$

ad esprimere che il rapporto delle funzioni corrispondenti è sul contorno della varietà 1 ; ed $\mathrm{i}$ sistemi $\varpi_{m}$ e $p_{m}$ si potranno dire equivalenti.

13. Riprendiamo la funzione analitica $v$ di $u$ definita dall'equazione (2), e fra i rami di questa funzione multiforme, distinguiamo quello (variabile anche da regione a regione del campo $u$ ) minimo in valore assoluto: indicandolo con $\varphi(u)$, la $\varphi(u)$ arrà un valore unico, senza discontinuità, per ogni valore di $u$ e potrà essere al più infinita (in casi eccezionali, $\$ 8$ ) in punti separati. Se ora consideriamo la funzione

$$
\frac{1}{v-\varphi(u)},
$$

questa sarà sviluppabile in serie convergente

$$
\Sigma \frac{\eta^{n}}{\varphi^{n+1}(u)}
$$

per tutte le coppie $u$ e $v$ tali che sia

$$
|\varphi(u)|>|v|
$$

(c) Journal de Creit.te, t. 73, $187 \mathrm{I}$. 
ma se poniamo $|v|=\alpha$, questa condizione è soddisfatta per $\mathrm{i}$ valori $u$ dell'area $E_{\alpha}(\S 9)$, e nel campo $E_{\alpha}^{\prime}$ la stessa serie è divergente: onde segue $(\S 12)$ che se $p_{m}(u)$ è un sistema di funzioni generate dalla funzione $T(u, v)$ i cui posti singolari soddisfanno alla (2), si avrà

$$
p_{m}(u) \sim \varphi^{-(m+1)}(u), \text { od anche } p_{m}(u) \sim \varphi^{-m}(u) .
$$

14. Si consideri come esempio l'equazione delle singolarità

$$
f(u, v)=u^{2}+v^{2}-1==0 .
$$

Da questa equazione risulta

e posto

$$
\varphi(u)=\sqrt{1-u^{2}}\left(^{*}\right), \quad \rho(u)=\left|\sqrt{1-u^{2}}\right|
$$

viene

$$
u=r(\cos t+i \operatorname{sen} t)
$$

$$
p^{4}=\left(1+r^{2}+2 r \cos t\right)\left(1+r^{2}-2 r \cos t\right),
$$

ossia le curve $C_{\alpha}$ sono cassinoidi aventi per fuochi i punti \pm 1 ; facendo $\alpha=0$ si ottengono i punti \pm 1 per punti singolari; essi corrispondono ai punti $0_{1}, 0_{2}$ della teoria generale. Ogni cassinoide $C_{a}$ divide il piano in due regioni: l'una $E_{\alpha}$ indefinita nella quale è $\rho>\alpha$, l'altra $E_{\alpha}^{\prime}$, con $\rho<\infty$, limitata e sconnessa se $\alpha<1$, semplicemente connessa per $\alpha>1$; per $\alpha=1$ la curva $C_{\alpha}$ ̀̀ la lemniscata.

Se $T(u, v)$ è la funzione generatrice di un sistema $p_{m}(u)$ colle singolarità nei posti $(u, v)$ soddisfacenti alla $(a)$, si avrà

Una serie

$$
p_{m}(u) \sim\left(1-u^{2}\right)^{-\frac{m}{2}}
$$

$$
\mathbf{\Sigma} c_{m} p_{m}(u)
$$

dove $\left(c_{m}\right)$ sia al contorno della varietà $\alpha$, converge fuori della cassinoide

$$
r^{4}-2 r^{2} \cos 2 t+1=\alpha^{4}
$$

essa converge fuori della lemniscata se $\left(c_{m}\right)$ è al contorno della varietà 1 , ed in tutto il piano eccettuati i due punti \pm 1 , se il gruppo $\left(c_{m}\right)$ i ologene.

Sia ora l'altra equazione di singolarità

$$
f(u, v)=1-2 u v+v^{2}=0
$$

() I due rami della funzione $v$ di u avendo lo stesso valore assoluto, si puó ritencre il segno + per $(u)$. 
che è quella delle funzioni sferiche. I punti $0_{n}$ si riducono all'infinito, per cui le funzioni $p_{n}(u)$ saranno intere qualunque sia la funzione generatrice. Dalla (b) risulta

$$
\varphi(u)=u \pm \sqrt{u^{2}-1}
$$

dove il segno \pm va preso in modo che si abbia la radice minore in valore assoluto, cioè $\rho(u)$ o $\propto$ deve essere costantemente minore dell' unità, e eguale ad 1 solo per $u$ reale e minore d'uno in valore assoluto. Posto $u=\xi+i n$, si ha per $\{\varphi(u t)=\alpha$ :

$$
\frac{n^{2}}{\frac{1}{4}\left(\alpha+\frac{1}{\alpha}\right)^{2}}+\frac{1}{\frac{1}{4}\left(\alpha-\frac{1}{\alpha}\right)^{2}}=1
$$

ossia il sistema delle curve $C_{\alpha}$ consta di ellissi coi fuochi nei punti \pm 1 . Variando * da 0 ad 1 , le ellissi variano dal cerchio di raggio infinito al segmento rettilineo da $+1 a-1$. L'area $E_{\alpha}$ è l'interno dell'ellisse $C_{\alpha}$, l'area $E^{\prime}$ * ne è l'esterno.

Se $T(u, v)$ è una funzione colle singolarità nei punti soddisfacenti alla (b), e generatrice di un sistema $p_{n}(u)$, si arrà

$$
p_{m}(u) \sim\left(u \pm \sqrt{u^{2}-1}\right)^{m}
$$

il segno \pm corrispondente al ramo maggiore dell' unità. Una serie

$$
\mathbf{\Sigma} c_{m} p_{m}(u)
$$

(in particolare una serie di funzioni sferiche) convergerà entro l'ellisse $C_{\alpha}$ se $\left(c_{m}\right)$ è sul contorno di una varietà $a<1$, cioè se la serie $\mathbf{\Sigma} c_{m} z^{m}$ converge in un cerchio $>1$; essa convergerà in tutto il piano se il gruppo $\left(c_{m}\right)$ è ologene.

III.

15 Fra i sistemi di funzioni considerati nel capo precedente, hanno speciale importanza quelli che danno lnogo ad uno sviluppo in serie della forma

$$
\mathbf{\Sigma} c_{m} p_{m}(u)
$$

per qualunque funzione analitica $f(u)$ regolare entro un campo abbastanza grande. Cio avviene per esempio quando sia possibile, sotto certe condizioni, lo sviluppo

$$
\frac{1}{y-x}=\mathbf{\Sigma} p_{n}(x) P_{n}(y)
$$


giacchè il teorema di CAdchr permette di dedurre da questa formola lo sviluppo in serie di funzioni $p_{m}(x)$ o $P_{m}(y)$ per qualunque funzione regolare in un campo abbastanza grande da conservare le condizioni di validità dello sviluppo (1).

Dato un sistema di funzioni $p_{m}(u)$, in generale diremo: " che un secondo " sistema di funzioni $P_{m}(u)$ è associato al sistema $p_{m}(u)$, se entro un determi" nato campo per le variabili $x$ ed $y$, la serie

$$
\mathbf{\Sigma} p_{m}(x) P_{m}(y)
$$

" converge assolutamente ed in egual grado ed ha per ogni coppia di valori $"$ di $x$ ed $y$, il valore

$$
\frac{1}{x-y} "
$$

16. Riprendiamo ora uno dei sistemi $p_{m}(u)$ quali si sono esaminati nel capo precedente: se esiste un sistema di funzioni $P_{m}(u)$ tali che sia $P_{m}(u) \sim \frac{1}{p_{m}(u)}$ la serie (2) convergerd per tutte le coppie $x$ ed $y$ tali che se $x$ è interna ad $E_{\alpha}, y$ sia interna ad $E_{\alpha}^{\prime}$.

Infatti preso $y$ in $E_{\alpha}^{\prime}$, si ha per il $\S 13$ :

$$
P_{m}(y) \sim \frac{1}{\eta^{m}(y)} \sim \varphi^{m}(y)
$$

ma si ha pure in $E_{\alpha}^{\prime \prime}$ :

$$
|\varphi(y)|<\alpha
$$

onde presa una quantità $\varepsilon$ positiva e piccola ad arbitrio, sarà

$$
\left|P_{m}(y)\right|<\alpha^{m}(1+\varepsilon)^{m}
$$

da cui risulta che la (2) converge per tutti i valori di $x$ compresi entro $E_{a(1+\varepsilon),}$ ed essendo $\varepsilon$ piccolo ad arbitrio, la (2) converge per tutti $\mathrm{i}$ valori interni ad $E_{a}$. Essa converge inoltre incondizionatamente, ed in egual grado rispetto ad $x$ entro $E_{\alpha}$ se si dà ad $y$ un valor fisso, e rispetto ad $y$ entro $E_{\alpha}^{\prime}$ se si dà un valor fisso ad $x$; quest' ultima parte si dimostrerebbe come a $\S 10$.

Ciò posto, supponiamo che questi sistemi $p_{m}(u)$ e $P_{m}(u)$ siano associati, ed inoltre che le curve $C_{\alpha}$ siano chiuse ed il campo da esse racchiuso sia semplicemente connesso: in tal modo, o la $E_{\alpha}$ sarà l'interno di $C_{\alpha}$ e la $E_{\alpha}^{\prime}$ l'esterno, ed allora le curve $C_{\alpha}$ andranno diminuendo al crescere di $\alpha$, perchè per $\alpha^{\prime}>\alpha$ la $E_{\alpha^{\prime}}$ è compresa in $E_{\alpha}$, o inversamente; il primo caso è per es. quello delle 
funzioni sferiche, il secondo è quello delle funzioni $\frac{1}{u^{m}}$. Dato il numero $\alpha$, si considerino altri due numeri vicini arbitrariamente ad $\alpha$,

$$
a^{\prime \prime}>x^{\prime}>x^{\prime}
$$

le curve $C_{\alpha^{\prime}}$ e $C_{a^{\prime \prime}}$ saranno vicinissime a $C_{\alpha}$, e $C_{o}^{\prime}$ sarà entro $E_{\alpha}$ e $C_{a^{\prime \prime}}$ entro $E_{\alpha^{\prime}}$. Preso ora $y$ lungo la $C_{\infty}$, per tutti i punti $x$ interni a al contorno del campo $E_{a^{\prime \prime}}$ la serie (2) sarà convergente in egual grado rispetto ad $y$ : se dunque $f(x)$ è una funzione regolare in tutto l'interno di $E_{\alpha}$, si avrà

$$
f(x)=\frac{1}{2 \pi i} \int_{C_{y, !}} \frac{f(y) d y}{y-x}=\mathbf{\Sigma} C_{m} p_{m}(x)
$$

dove

$$
C_{m}=\frac{1}{2 \pi i} \int_{C_{y \prime}} f(y) P_{m}(y) d y .
$$

In modo analogo si otterrebbero sviluppi in serie di funzioni $P_{m}$ per una funzione regolare entro un campo $E_{\alpha}$, ed in serie di funzioni $p_{n}$ e $P_{m}$ per una funzione regolare in un campo connesso compreso fra due curve $C_{\alpha}$ e $C_{\beta}$ del sistema.

17. Dato il sistema di curve $C_{*}$ ed $\mathrm{i}$ sistemi associati di funzioni $p_{m}$ e $P_{m}$ di cui al paragrafo precedente, e supponendo di più le funzioni $p_{m}$ intere, si abbiano i punti

$$
a_{1}, \quad a_{2}, \quad a_{3}, \ldots \quad a_{n}, \ldots
$$

in numero infinito aventi per limite una linea $C_{t}$ del sistema e d'altronde tutti contenuti entro il campo $E_{f}$ o sul contorno, ed altrettante funzioni intere

$$
G_{1}(x), \quad G_{2}(x), \ldots \quad G_{n}(x), \ldots
$$

Si domanda se esisterà una funzione analitica $F(x)$ che in tutto jl campo $E_{0}$ sia regolare, eccettuati i punti $a_{n}$, nei quali essa abbia rispettivamente le stesse singolarità delle $G_{n}\left(\frac{1}{x-a_{n}}\right)$.

A quest'uopo, consideriamo le linee del sistema $C_{c}$ :

$$
C_{\alpha\left(a_{1}\right)}, \quad C_{\alpha\left(a_{2}\right)}, \ldots \quad C_{\alpha\left(a_{n}\right)}, \ldots
$$

passanti per i punti

$$
a_{1}, \quad a_{2}, \ldots \quad a_{n}, \ldots
$$


rispettivamente, e curve parallele

$$
C_{1}^{\prime}, \quad C_{2}^{\prime}, \ldots \quad C_{n}^{\prime}, \ldots
$$

tali che $C_{n}^{\prime}$ sia nel campo $E_{\alpha\left(a_{n}\right)}$ parallela a $C_{o\left(a_{n}\right)}$ e ad una distanza $\delta_{n}$ contata sulle normali per modo che

$$
\lim _{n=\infty} \delta_{n}=0
$$

indichiamo infine con $F_{n}$ il campo che rimane da $E_{a\left(a_{n}\right)}$ togliendovi l'anello compreso fra $C_{\alpha\left(a_{n}\right)}$ e $C_{n}^{\prime}$.

La funzione $G_{n}\left(\frac{1}{x-a_{n}}\right)$ essendo regolare entro tutto il campo $E_{\alpha\left(a_{n}\right)}$, si avrà $(\S 16)$

$$
G_{n}\left(\frac{1}{x-a_{n}}\right)=\sum_{m=0}^{\infty} c_{n, m} p_{m n}(x)
$$

si prenda ora un sistema di numeri positivi

$$
\varepsilon_{1}, \quad \varepsilon_{2}, \ldots \quad \varepsilon_{n}, \ldots
$$

tali che $\sum \varepsilon_{n}$ sia convergente, e si faccia $\mu_{n}$ tanto grande che sia entro tutto $F_{n}$

$$
\left|\sum_{m=\mu_{n}}^{\infty} c_{n, m} p_{m}(x)\right|<\varepsilon_{n},
$$

il che è possibile.

Ponendo

$$
\varphi_{n}(x)=G_{n}\left(\frac{1}{x-u_{n}}\right)-\sum_{m=0}^{\mu_{n}} c_{n, m} p_{m}(x)
$$

questa sarà una funzione analitica regolare in tutto il piano, tranne il punto $a_{n}$ nel quale essa diventa singolare come la $G_{n}$.

Si consideri infine la funzione

$$
F(x)=\sum_{n=1}^{\infty} \varphi_{n}(x)
$$

Per un punto $x$ interno ad $C_{\rho}$ e che non sia uno dei punti $a_{n}$, si può sempre prendere $N$ tanto grande che tutti i punti $a_{n}$ per $n>N$ siano fuori dell'area $E_{a(x)}$ relativa a quel punto. Si avrà dunque

$$
F(x)=\varphi_{1}(x)+\varphi_{2}(x)+\cdots \varphi_{N}(x)+\sum_{n=N+1}^{\infty} \varphi_{n}(x),
$$

ed essendo $x$ interno a tutte le aree $F_{n}$ per $n>N$, verrà

$$
\left|\sum_{N+1}^{\infty} \varphi_{n}(x)\right|<\sum_{N+1}^{\infty} \varepsilon_{n}
$$


cioè la $F(x)$ è finita e regolare nell' intorno di $x$. Se invece $x$ è uno dei punti $a_{n},\left(x=\alpha_{*}\right)$ si dimostra in modo analogo che

$$
F(x)-\varphi_{\nu}(x)
$$

è regolare, onde $F(x)$ è singolare in $a_{\nu}$ come $G\left(\frac{1}{x-u_{\nu}}\right)$.

Se i punti dati $a_{n}$ tendessero alla linea $C_{\rho}$ per modo che ogni punto della $C_{\rho}$ sia punto limite per il sistema dei punti $a_{n}$, la funzione $F(x)$ costruita come si è detto avrebbe l'area $E^{\prime}$ per spazio lacunare.

Lo stesso problema si può anche risolvere in modo analogo senza supporre le funzioni $p_{m}(x)$ intere; le funzioni $\varphi_{n}(x)$ e quindi la $F(x)$ sarebbero allora singolari anche nei posti già detti $0_{1}, 0_{2}, \ldots 0_{n}, \ldots$ a $\S 6$.

\section{IV.}

18. Lo sviluppo di una funzione analitica in serie procedente per un sistema dato di funzioni $p_{m}(x)$ si ottiene generalmente per mezzo di due metodi ben noti, che si possono adoperare:

11 primo, quando esiste uno sviluppo di $\frac{1}{y-x}$ in serie di funzioni $p_{m}(x)$; in tal caso lo sviluppo della funzione analitica si ottiene applicando il teorema di CAUChY;

Il secondo, quando esiste un secondo sistema di funzioni $q_{n}(x)$, una costante $C$ ed una linea $\gamma$ nel piano, tali che sia

$$
\int_{(y)} p_{m}(x) q_{n}(x)=\left\{\begin{array}{l}
C \text { se } m=n \\
0 \text { se } m=n
\end{array}\right.
$$

ed in tal caso lo sviluppo della funzione si ottiene con un processo di coefficienti indeterminati.

In questo capitolo e nel seguente mi propongo di accennare ad un altro metodo atto a risolvere lo stesso problema e che presenta il vantaggio di mettere in chiaro come le relazioni per mezzo d'integrali definiti che servono nel secondo dei metodi ricordati altro non siano che passaggi al limite di relazioni notissime elementari. Questo metodo presentandosi però come applicazione di considerazioni più generali sui gruppi di numeri, queste verranno esposte per prime. 
19. Abbiasi un gruppo di numeri doppiamente infinito

$$
\left(a_{m, n}\right) \quad\left\{\begin{array}{l}
m=0,1, \ldots \infty \\
n=0,1, \ldots \infty
\end{array}\right.
$$

soggetto alla condizione che sia possibile determinare tre numeri positivi $A$, $r, s$ tali che per ogni valore di $m$ e di $n$ sia

$$
\left|a_{m, n}\right|<\frac{A}{r^{m} g^{n}} \text {. }
$$

È chiaro che determinata una coppia di numeri $r$ ed $s$, ne esistono infinite altre che permettono di soddisfare alla disuguaglianza (2).

Il gruppo (1) potendosi scrivere

$$
\begin{array}{cccc}
a_{0,0} & a_{0,1} & a_{0,2} \ldots & a_{0, n} \ldots \\
a_{1,0} & a_{1,1} & a_{1,2} \ldots & a_{1, n} \ldots \\
\ldots & \ldots & \ldots & \ldots \\
a_{m 0} & a_{m, 1} & a_{m, 2} \ldots & a_{m, n} \ldots
\end{array}
$$

sarà lecito dire che il primo indice esprime la linea ed il secondo la colonna cui appartiene un numero od elemento del gruppo.

Se $\left(x_{n}\right)$ è un gruppo posto nell' interno della varietà $s$, le serie

$$
\sum_{n=1}^{\infty} a_{m, n} x_{n}
$$

saranno convergenti incondizionatamente per ogni valore di $m$, come si verifica immediatamente, ed indicando con $y_{m}$ il valore delle serie (3) corrispondente all'indice $m$ e con $M$ un numero positivo finito ed indipendente da $m$, sarà

$$
\mid y_{m i}<\frac{M}{r^{m}}
$$

cioè il gruppo $\left(y_{m}\right)$ sarà interno 0 al contorno della varietà $\frac{1}{r} \cdot$ Adunque, ad ogni gruppo $\left(x_{n}\right)$ interno alla varietà $s$, le formole

$$
y_{m}=\sum_{n=0}^{\infty} a_{m, n} x_{n}
$$

fanno corrispondere un gruppo determinato interno o al contorno della varietà $\frac{1}{r}$. 
Analogamente, le formole

$$
y_{n}^{\prime}=\sum_{m=0}^{\infty} a_{m, n} x_{m}^{\prime}
$$

farebbero corrispondere ad ognj gruppo $\left(x^{\prime} m\right)$ irterno alla varietà $r$, un gruppo determinato interno o al contorno della varietà $\frac{1}{s}$.

20. Supponiamo ora che insieme alle $\left(a_{m}, n\right)$ esista un secondo gruppo doppiamente infinito

$$
(\alpha m, n)
$$

per il quale sia possibile determinare tre numeri positivi $B, \rho, \sigma$ tali che sia

$$
\left|\alpha_{m} \cdot n\right|<\frac{B}{p^{m} \sigma^{n}}
$$

e sia per ogni valore di $n$

$$
\sum_{m=0}^{\infty} a_{m, n} \alpha_{m, \nu}=\left\{\begin{array}{l}
0 \text { per } n \geq \nu \\
1 \text { per } n=\nu
\end{array}\right.
$$

infine fra le infinite determinazioni di $r, s, \rho$ e $\sigma$ ve ne siano che soddisfacciano alle condizioni

$$
r_{\rho}>1, \quad s \sigma>1 \text {. }
$$

Sotto queste ipotesi, dico:

$1 .^{\circ}$ Che dalle equazioni $\left(3^{\prime}\right)$ si può ricavare

$$
x_{n}=\sum_{m=0}^{\infty} \alpha_{m, n} y_{m},
$$

$2 .^{\circ}$ Che fra le $a_{i n, n}$ e le $\alpha_{m, n}$ passa l'altra relazione

$$
\sum_{n=0}^{\infty} a_{m, n} \alpha_{\mu, n}=\left\{\begin{array}{l}
0 \text { per } m \geq \mu . \\
1 \text { per } m=\mu .
\end{array}\right.
$$

a) Si ponga infatti

$$
X_{n}=\sum_{m=0}^{\infty} \alpha_{m, n} y_{m}:
$$

la serie del secondo membro è convergente incondizionatamente se si ha

$$
\left|y_{m}\right|<\frac{M}{r^{m}}
$$

ed indicando con $N$ una quantità finita, viene

$$
\left|X_{n}\right|<\frac{N}{\sigma^{n}},
$$


e per la (6)

$$
X_{n}<N s^{n}
$$

ossia le $X_{n}$ sono nell' interno o al contorno della varietà s. Ma si ha altresi, ponendo per le $y_{m}$ i loro valori $\left(3^{\prime}\right)$ :

$$
X_{n}=\sum_{m=0}^{\infty} \alpha_{m, n} \sum_{y=0}^{\infty} a_{m, \nu} x_{\nu}
$$

ora un termine di questa serie doppia è in valore assoluto

$$
<\frac{A B s^{\prime \prime}}{(r \rho)^{m} \sigma^{n} s^{y}}
$$

cioè la serie doppia ha $\mathrm{i}$ suoi termini minori in valore assoluto della serie a termini positivi

$$
\frac{A B}{c^{n}} \sum_{m, 2} \frac{1}{(r p)^{m}}\left(\frac{s^{\prime}}{s}\right)^{y}
$$

che è convergente; per cui nel secondo membro della (9) i termini si possono ordinare come si vuole e viene

e per le (5)

$$
X_{n}=\sum_{\nu=0}^{\infty} x_{\nu}\left(\sum_{m=0}^{\infty} \alpha, n, n a_{m, \nu}\right)
$$

$$
X_{n}=x_{n}
$$

Dunque sotto le ipotesi fatte, le $\left(3^{\prime}\right)$ si possono riguardare come un sistema di infinite equazioni lineari con infinite incognite, di cui le (7) danno la soluzione. doppie della forma

b) Nelle (3') poniamo per $x_{n}$ i loro valori (7), e vengono le serie

$$
\sum_{n} a_{m, n} \sum_{\mu} \alpha_{\mu, n} y_{\mu}
$$

ora un termine di questa serie è in valore assoluto

$$
<\frac{A B}{r^{m}} \frac{1}{(s \tau)^{n}} \frac{1}{(r p)^{m}}
$$

ma la serie di questi termini positivi ̀̀

$$
<\frac{A B}{r^{m}} \sum_{n, p} \frac{1}{(s \sigma)^{n}(r \rho)^{\mu}}
$$

cioè è convergente, per cui la (10) sard̀ convergente assolutamente ed i suoi termini potendosi ordinare come si vuole, si potrà scrivere

$$
y_{m}=\sum_{\mu} y_{\mu}\left(\sum_{n} a_{m, n} \alpha_{\mu, n}\right)
$$


Ma le $y_{m}$ sono numeri arbitrarî soggetti alla sola condizione

$$
\left|y^{n}\right|<\frac{1}{r^{m}}
$$

prendiamo pertanto

$$
y_{n}=n^{n}
$$

dove $\eta$ è un numero complesso arbitrario e tale che

$$
|n|<\frac{1}{r}
$$

e viene dallo sviluppo precedente per ogni valore di $\eta$ soggetto a questa condizione:

$$
r^{m}=\sum_{\mu^{m}} n^{\mu} \sum_{n} a_{m, n} \alpha_{\mu, n}
$$

il che è impossibile, a meno che non sia

$$
\sum_{n} a_{m, n} \alpha_{\mu, n}=\left\{\begin{array}{l}
0 \text { per } m>\mu \\
1 \text { per } m=\mu .
\end{array}\right.
$$

21. Due sistemi di numeri che soddisfanno alle relazioni

$$
\sum_{m} a_{m, n} \alpha_{m, \nu}=\left\{\begin{array}{l}
0 \text { per } n \geq \nu \\
1 \text { per } n=\nu,
\end{array} \quad \sum_{n} a_{m, n} \alpha_{\mu, n}=\left\{\begin{array}{l}
0 \text { per } m \geq \mu \\
1 \text { per } m=\mu
\end{array}\right.\right.
$$

(le serie dei primi membri essendo d'altronde convergenti) si diranno associati; sono dunque associati i sistemi $a_{a, n}$ ed $a_{m, n}$ del paragrafo precedente, sotto le ipotesi fatte al principio del paragrafo stesso.

Sotto quello ipotesi, nello stesso modo che da

si ricava

$$
\left.\begin{array}{l}
y_{m}=\sum_{n} \alpha_{m, n} x_{n} \\
x_{n}=\sum_{m} \alpha_{m, n} y_{m}
\end{array}\right\}
$$

$\operatorname{cosi} \mathrm{da}$

si ricaverd

$$
\begin{aligned}
& u_{n}=\sum_{m} a_{m, n} v_{m} \\
& v_{m}=\sum_{n} \alpha_{m, n} u_{n} .
\end{aligned}
$$

prendendo le $u_{n}$ nell'interno della varietà $\sigma^{\prime}<\sigma$, e le $v_{n}$ essendo di conseguenza nell' interno o al contorno della varietì $\frac{1}{0}$.

E chiamando le $(b)$ formole di trasformazione inversa delle $(a)$, viene clie 
sotto le stesse ipotesi se in una serie

$$
\sum u_{n} x_{n}
$$

sulle $x$ si fa la trasformazione (a) e sulle $u$ la trasformazione inversa, la serie si trasformerà in

infatti la serie

$$
\sum v_{m} y_{m}
$$

$$
\sum_{n=0}^{\infty} u_{n} \sum_{m=0}^{\infty} \alpha_{n}, n y_{m}
$$

ha $\mathrm{i}$ suoi termini minori in valore assoluto di quelli della serie a termini positivi

$$
\sum_{n} \sigma_{n}^{\prime} \sum_{m} \frac{B}{\rho^{n, n}} \cdot \frac{1}{r^{m}}=B \sum_{m, n} \frac{1}{(r \rho)^{m}}\left(\frac{\sigma^{\prime}}{\sigma}\right)^{n}
$$

che è convergente; adunque la (11) si può ordinare come si vuole, e viene d'una parte

$$
\sum_{n=0}^{\infty} u_{n}\left(\sum_{m=0}^{\infty} \alpha_{m, n} y_{m}\right)=\mathbf{\Sigma} u_{n} x_{n}
$$

dall'altra

$$
\sum_{m=0}^{\infty} y^{m}\left(\sum_{n=0}^{\infty} \alpha_{m}, n u_{n}\right)=\sum y^{m} v_{n} \text {. }
$$

Da queste considerazioni risulta che i gruppi

$$
\left(\alpha_{m, n}\right) \text { ed }(\alpha, m, n)
$$

sotto le ipotesi dei $\S \S 19$ e 20 , si comportano come il sistema degli elementi di un determinante $D$ col sistema degli elementi reciproci divisi per $D$. Le formole $(a)$ e $(b)$ estendono alle varietà (spazî ad $\infty$ dimensioni) i concetti della trasformazione lineare degli spazî di $n$ dimensioni.

22. In certi casi si può determinare il gruppo associato di un gruppo dato.

a) Se si ha una funzione analitica $y$ di $x$, nulla del prim'ordine per $x=0$ e regolare in un certo intorno $R$ di $x=0$,

$$
y=a_{1,1} x+a_{1,2} x^{2}+\cdots a_{1, n} x^{n}+\cdots
$$

è noto che se ne può dedurre in un certo intorno $R^{\prime}$ di $y=0$

$$
x=\alpha_{1,1} y+\alpha_{2,1} y^{2}+\cdots \alpha_{n, 1} y^{n}+\cdots
$$

Coi noti teoremi della moltiplicazione delle serie se ne deduce

$$
\begin{aligned}
& y^{n}=\sum_{r=0}^{\infty} a_{n, n+r} x^{n+r} \\
& x^{n}=\sum_{r=0}^{\infty} a_{n+r}, n y^{n+r} ;
\end{aligned}
$$


ora i gruppi $(a m, n),(a m, n)$ sono associati. Infatti ponendo nella (14) le espressioni di $x^{n+r}$ date dalle (15) osserviamo che per $|y|$ abbastanza piccolo viene $|x|<R$ e quindi la serie di funzione di $y$ del secondo membro della (14) ̀̀ convergente in egual grado; si potrà quindi ordinarla rispetto alle potenze di $y$, ed eguagliando nei due membri i coefficienti di $y^{n}$, verrà

$$
\begin{gathered}
a_{n, n} \alpha_{n, n}=1 \\
a_{n n \alpha_{n, n}}+a_{n, n+1} \alpha_{, n, n+1}+\cdots+a_{n, \nu, \nu}=0 ;
\end{gathered}
$$

queste sono le relazioni (5) o (8) del paragrafo precedente.

b) Se il gruppo $\left(a_{m n}\right)$ è tale che sia

$$
a_{m, n}=0 \text { per } n>m
$$

gli elementi del gruppo associato dovranno essere nulli per $n<m$ : si ha cosi lo specchio

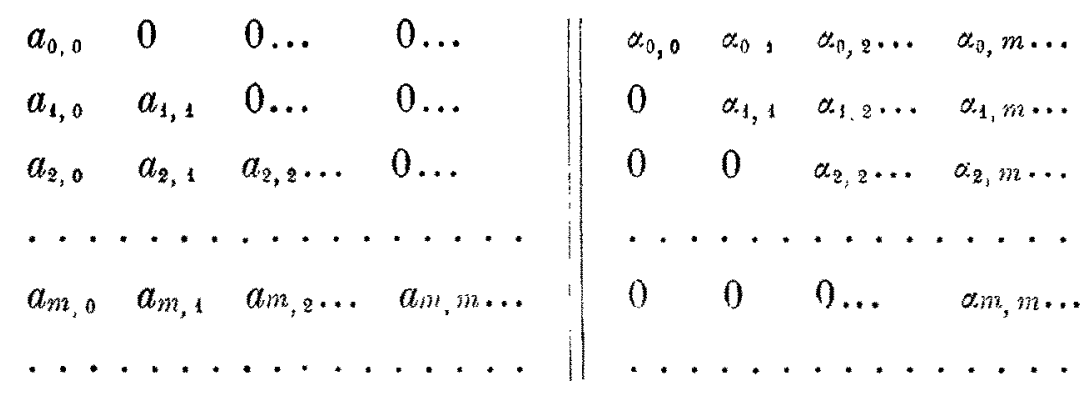

e le relazioni (5) e (8) del paragrafo precedente divengono:

$$
\begin{aligned}
a_{m, m} \alpha_{m, m}=1 & \\
a_{m, \mu} \alpha_{\mu, \mu}+a_{m, n+1} \alpha_{\mu, \mu+1}+\cdots+\alpha_{n, m} \alpha_{\mu, m}=0 & (\mu<m) \\
a_{n, n} \alpha_{n, \nu}+a_{n+1, n} \alpha_{n+1, \nu}+\cdots+\alpha_{\nu, n} \alpha_{\nu, \nu}=0 & (\nu>n) .
\end{aligned}
$$

I numeri $\alpha_{m, n}$ si possono determinare di mano in mano formando i determjnanti d'ordine $n$ colle prime $a_{n_{n} n}$ e calcolando gli elementi reciproci, indi dividendo per il determinante.

In questo caso, si semplificano le condizioni date al $\$ 20$; per es. se si ha

ed

$$
a_{m, m}=1
$$

$$
\left|a_{r i+x, m}, m\right|<\frac{A}{s^{\prime \prime}}
$$


saranno valide le formole di trasformazione $(a)$ e $(b)$ del $\$ 21$; ciò si trova dimostrato in altro mio lavoro $\left(^{*}\right)$.

c) Al caso precedente si riducono pure i gruppi tali che sia

$$
a_{m, n}=0 \text { per } n<m \text {; }
$$

un tal gruppo si otterrebbe dal precedente mutando le linee in colonne e inversamente.

$\mathrm{V}$.

23. Applichiamo ora le considerazioni precedenti a sistemi di funzioni; riprendiamo le ipotesi del $\S 20$, supponiamo cioè di avere due gruppi $\left(a_{m}, n\right)$, $\left(\alpha_{m, n}\right)$ tali che sia

$$
\begin{array}{cl}
\left|a_{m, n}\right|<\frac{A}{r^{m} s^{n}}, & \left|\alpha_{m, n}\right|<\frac{B}{\rho^{m} \sigma^{n}}, \\
r \rho>1, & s \sigma>1
\end{array}
$$

dove uno dei sistemi (3) è conseguenza dell' altro.

Un sistema $\left(a_{m, n}\right)$ soddisfacente alla (1) ci sarà dato ogniqualvolta arremo una funzione di due variabili $u, v$ regolare in un intorno del posto $(u=0$, $v=0$ ); se sarà infatti

$$
T(u, v)=\Sigma a_{m, n} u^{m} v^{n}
$$

$r, s$ saranno numeri qualunque positivi inferiori ai raggi di convergenza nei piani $u$ e $v$. La $T(u, v)$ sarà funzione generatrice dei due sistemi di funzioni

$$
\begin{aligned}
& p_{m}(v)=\sum_{n} a_{m, n} v^{n} \\
& q_{n}(u)=\sum_{m} a_{m, n} u^{m},
\end{aligned}
$$

le prime regolari tutte entro il cerchio di raggio $s$, le seconde entro il cerchio di raggio $r$.

Il sistema associato definirà una seconda funzione di due variabili

$$
\Theta(u, v)=\sum \alpha m, n u^{m} v^{n}
$$

(') Sopra alcuni sviluppi in serie per funzioni analitiche. Memorie dell' Accademia di Bologna, serie IV, t. 3 . 
che genererà $\mathrm{i}$ due sistemi di funzioni di una variabile

$$
\begin{aligned}
& P_{m}(u)=\sum_{n} \alpha_{m, n} v^{n} \\
& Q_{n}(v)=\sum_{m} \alpha_{m}, n u^{m}
\end{aligned}
$$

regolari rispettivamente entro $\mathrm{i}$ cerchi di raggio $\sigma$ e $\rho$.

24. "Ogni funzione $f(x)$ regolare entro un cerchio di centro $x=0$ e di n raggio maggiore di $s$, è sotto le ipotesi del paragrafo precedente, svilup" pabile in serie di funzioni $p_{m}(x)$."

Sia

$$
f(x)=\Sigma A_{\nu} x^{y}
$$

la funzione data; essendo essa regolare entro un cerchio maggiore di $s$, il gruppo $\left(A_{\nu}\right)$ sarà interno alla varietà $\frac{1}{S}$, e per le $(2)$, alla varietà $\sigma$.

Ne segue $(\$ 19)$ che ponendo

$$
C_{m}=\sum_{n} \alpha . m, n A_{n}
$$

le $C_{m}$ avranno valori determinati e tali che sia

$$
\left|C_{m}\right|<\frac{M}{p^{n t}}
$$

e dalle formole (4) risulterà $(\$ 20)$

Formando la

$$
A_{n}=\sum_{m} a_{m, n} C_{n} \text {. }
$$

$$
f(x)=\sum A_{\nu} x^{\nu}=\sum_{\nu} x_{m}^{\nu} \sum_{m, \nu} a_{n}
$$

questa serie doppia ha per $|x| \leqslant s^{\prime}<s$ i suoi termini minori in valore assoluto di quelli della serie convergente e a termini positivi

$$
A M \sum_{m, \nu}\left(\frac{s^{2}}{s}\right)^{v} \frac{1}{\left(r_{p}\right)^{m}}
$$

per cui $i$ suoi termini si potranno ordinare come si vuole, ed in particolare si potrà scrivere

$$
f(x)=\underset{m}{\mathbf{Z}} C_{m} \sum_{\nu} a_{n, v} x^{y}=\underset{m}{\mathbf{Z}} C_{m} p_{m}(x)
$$

c. d. d.

25. Sotto le stesse ipotesi è possibile determinare un sistema di funzioni associate $(\S 15)$ di $p_{m}(x)$ e quindi uno sviluppo della forma (5) applicando il 
teorema di $\mathrm{C}_{\mathrm{AUCH}}$. Si ha infatti $(\S 21)$ che

$$
\Sigma u_{n} x_{n}=\Sigma v_{m} y_{m} \text {. }
$$

Se si limitano convenientemente le varietà cui appartengono i gruppi

$$
\left(u_{n}\right), \quad\left(x_{n}\right), \quad\left(v_{m}\right), \quad\left(y_{m}\right)
$$

e se questi gruppi si legano dalle relazioni

ponendo ora

$$
\begin{aligned}
& y_{m}=\sum_{n} a_{m, n} x_{n} \text { equivalente a } x_{n}=\mathbf{\sum} \alpha_{m, n} y_{m} \\
& u_{n}=\sum_{m} a_{m, n} v_{m} \quad n \quad \eta \quad v_{m}=\sum_{n} \alpha_{m, n} u_{n} ;
\end{aligned}
$$

sotto le condizioni

varrà l'eguaglianza

$$
\begin{array}{ll}
x_{n}=x^{n}, & y_{m}=p_{m}(x)=\sum_{n} a_{m, n} x^{n} \\
u_{n}=x_{n}^{\prime}, & v_{m}=P_{m}\left(x^{\prime}\right)=\sum_{n} \alpha_{m, n} x^{\prime n}
\end{array}
$$

e ponendo

$$
|x|<s, \quad\left|x^{\prime}\right|<\sigma, \quad\left|x x^{\prime}\right|<1
$$

si ha

$$
\frac{1}{1-x x^{\prime}}=\mathbf{\Sigma} p_{m}(x) P_{m}\left(x^{\prime}\right)
$$

$$
\frac{1}{y} P_{m}\left(\frac{1}{y}\right)=\omega_{m}(y)
$$

$$
\frac{1}{y-x}=\sum_{m} p_{m}(x) \varpi_{m}(y)
$$

formola valida almeno per $|x|<s$ ed $|y|>\frac{1}{\sigma}$; inoltre avendosi sotto le stesse condizioni

$$
\left|p_{m}(x)\right|<\frac{M}{r^{m}}, \quad\left|P_{m}\left(\frac{1}{y}\right)\right|<\frac{M^{\prime}}{\rho^{m}}
$$

con $r \rho>1$, ne risulta la convergenza assoluta ed in egual grado della serie (6) rispetto alle due variabili.

Risulta dalla (6) che se $f(x)$ è una funzione regolare entro un cerchio di centro 0 e di raggio maggiore di $s$, si avrà

$$
f(x)=\frac{1}{2 \pi i} \mathbf{\Sigma} p_{m}(x) \int f(y) \varpi_{m}(y) d y=\mathbf{\Sigma} C^{\prime}{ }_{m} p_{m}(x)
$$

l'integrazione essendo estesa ad una curva chiusa compresa entro il cerchio di convergenza di $f(x)$ ma esterna al cerchio di centro 0 e di raggio $s$. 
26. Tanto nello sviluppo (5) che nello sviluppo (7) i coefficienti $C_{m}$ e $C_{m}^{\prime}$ sono interni alla varietà $r$ infatti nel primo si ha

e nel secondo,

$$
\left|C_{m}\right|<\sum_{n}\left|A_{n} \alpha_{m}, n\right|<\frac{B}{\rho^{m}} \mathbf{\Sigma} \frac{1}{(s \sigma)^{n}}
$$

$$
\left|C_{m}^{\prime}\right|<\frac{L M^{\prime}}{p^{m}}
$$

essendo $L$ il massimo della funzione $f(x)$ nell' interno della curva d'integrazione; dico che essi coincidono, cioè che non può esistere uno sviluppo dello zero

$$
\mathbf{\Sigma} c_{m} p_{m}(x)=0
$$

nel quale $\left(c_{m}\right)$ sia interno alla varietà $r$ ed inoltre convergente in egual grado in un campo che comprenda il punto zero, sia pure arbitrariamente piccolo. Si potrebbe infatti in tale ipotesi sviluppare la (8) in serie di potenze di $x$ e si sa che dovrebbero essere nulli tutti i coefficienti, per cui

$$
\sum_{m=0}^{\infty} c_{m} a_{m, v}=0 \quad(r=0,1, \ldots \infty)
$$

considerando ora la serie doppia

essendo per ipotesi

$$
S_{\mu}=\sum_{\nu=0}^{\infty} \sum_{m=0}^{\infty} c_{m} a_{m, \nu} \alpha_{\mu, \nu}
$$

$$
\left|c_{m}\right|<H r^{\prime m}, \quad r^{\prime}<r
$$

i termini di questa serie sono in valore assoluto minori dei termini della serie

$$
A B H \underset{v, m}{\mathbf{\Sigma}} r^{\prime m} \frac{1}{r^{m} s^{\nu}} \frac{1}{\rho^{\mu} \sigma^{\nu}}=\frac{A B H}{\rho^{\mu}} \sum_{\nu, m}\left(\frac{r^{\prime}}{r}\right)^{m} \frac{1}{(s \sigma)^{\nu}}
$$

certamente convergente: per oui i termini della $S$ si potranno ordinare come si vuole e si avrà d'una parte

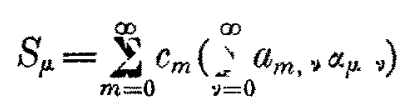

e per le relazioni (8) del $\S 20$ :

d'altra parte

$$
S_{\mu}=c_{\mu}
$$

$$
S_{\mu}=\sum_{\nu=0}^{\infty} \alpha_{\mu, \nu}\left(\sum_{m=0}^{\infty} c_{m} a_{m, \nu}\right)
$$


ossia, per le (9)

$$
S_{\mu}=c_{\mu}=0
$$

onde non può esistere uno sviluppo della forma (8) nel quale $\mathrm{i}$ coefficienti siano diversi da zero. Ne risulta l'identità fra gli sviluppi (5) e (7) della $f(x)$, e quindi

$$
\sum_{n} \alpha_{m, n} A_{n}=\frac{1}{2 \pi i} \int f(y) \omega_{m}(y) d y .
$$

Le considerazioni fatte sulle funzioni $p_{m}(x)$ nei due paragrafi precedenti si possono ripetere per le $q_{n}(x)$ definite da

$$
\sum_{m=0}^{\infty} a_{m, n} x^{m}
$$

Je cui associate sono

$$
\frac{1}{y} Q_{n}\left(\frac{1}{y}\right)=\sum_{m=0}^{\infty} \alpha_{m, n} \frac{1}{y^{m+1}} .
$$

27. Dalle posizioni

risulta

$$
\begin{aligned}
& T(u, v)=\mathbf{\Sigma} \alpha_{m, n} u^{m} v^{n} \\
& \Theta(u, v)=\mathbf{\Sigma} \alpha_{m, n} u^{m} v^{n}
\end{aligned}
$$

$$
p_{m}(v)=\frac{1}{2 \pi i} \int_{(u)} \frac{T(u, v) d u}{u^{m+}}, \quad q_{n}(u)=\frac{1}{2 \pi i} \int_{(v)} \frac{T(u, v) d v}{v^{n+1}}
$$

l'integrazione essendo estesa la prima volta ad un cerchio di raggio $<r$ nel piano $u$, la seconda volta nel piano $v$ ad un cerchio di raggio $<s$; da queste

Analogamente

$$
a_{m, n}=-\frac{1}{4 \pi^{2}} \int_{(u)} \int_{(\sigma)} \frac{T(u, v) d u d v}{u^{m+1} v^{n+1}} .
$$

$$
P_{m}(v)=\frac{1}{2 \pi i} \int_{(u)} \frac{\Theta(u, v) d u}{u^{m+1}}, \quad Q_{n}(u)=\frac{1}{2 \pi i} \int \frac{\Theta(u, v) d v}{v^{n+1}} .
$$

Si ponga ora nella (7)

e verrà

$$
f(x)=p_{m}(x)
$$

$$
p_{m}(x)=\Sigma C_{\mu}^{(m)} p_{\mu}(x)
$$

ma poichè non possono esistere sviluppi dello zero per funzioni $p_{\mu}(x)$, sard

$$
C_{m}^{(m)}=\left\{\begin{array}{l}
0 \text { per } \mu \gtrless m \\
1 \text { per } \mu=m
\end{array}\right.
$$


e ponendo per questi coefficienti le Ioro espressioni in forma d'integrali

$$
\int_{(C)} \varpi_{\mu}(y) p_{m}(y) d y=\left\{\begin{array}{c}
0 \text { per } \mu=m \\
2 \pi i \text { per } \mu=m
\end{array}\right.
$$

l'integrazione essendo estesa come a $\S 25$. Una formola analoga vale per le $q_{n}(x)$. Queste formole (12) sono l'espressione in forma d'integrale definito delle formole (5) e (8) del $\S 20$, e come tali si possono riguardare come una estensione delle relazioni fra gli elementi di un determinante ed i rispettivi elementi reciproci.

Bologna, aprile 1883. 


\section{N D I C E.}

Intropuzione. Richiamo di quelle proprietà delle scrie di funzioni sferiche che hanno suggerito la presente Memoria. Divisione di questo lavoro . . . . . . . . . . Pag. 11

Cap. I. Definizioni e considerazioni sui gruppi di numeri $(\$ \$ 1-4)$ ed $i$ sistemi di funzioni $(\$ 5)$. . . . . . . . . . . . . . . . . . . . \$13

Cap. II. Funzioni di due variabili come generatrici di sistemi di funzioni di una variabile (\$ 6). Campi di convergenza delle sevie formate colle funzioni di un tal sistema (\$§ 7-10). Varî sistemi di funzioni equivalenti, cioè le cui serie convergono negli stessi campi (\$S 11-12). Fra i sisteni equivalenti ve n'ha uno che consta dellc potenze di uno stesso ramo di funzione analitica $(\$ 19)$. Esempi: un caso in cui le curve di convergenza sono cassinoid; un caso in cui sono ellissi (\$ 14) .

Cap. III. Definizione e alcune proprietà dei sistemi associati di funzioni (\$§ 15้-16). Risoluzione del problema di Mrtrag-Lefreen per un'area sempliccmente commessa o costruzione di funzioni con spazî lacunari (\$ 17) . . . . . . . . . . .

Cap. IV. Metodi che servono ordinariamente allo sviluppo di una funzione in serie di determinata forma (\$ 18). Teoria dei gruppi associati, analogie colla teoria dei determinanti (§ 19-21), Casi particolari (\$ 22) . . . . . . . . . . . . . . .

Cap. V. Applicazione della teoria dei gruppi associati allo sviluppo di nua funzione in serie ( $8 \$ 23-25)$. Unicita degli sviluppi ottenuti in talo modo ( 26$)$. Lspressione in forma d'integrale delle relazioni fra $i$ gruppi associati (s 27) . 